\title{
Impact of Electronic Polarization on Preformed, $\beta$-Strand Rich Homogenous and Heterogenous Amyloid Oligomers
}

\author{
Kelsie M. King ${ }^{1}$, Amanda K. Sharp ${ }^{1}$, Darcy S. Davidson ${ }^{2}$, Anne M. Brown ${ }^{1,2,3 *}$, Justin A. Lemku1 ${ }^{2 *}$
}

${ }^{1}$ Program in Genetics, Bioinformatics, and Computational Biology, ${ }^{2}$ Department of Biochemistry, ${ }^{3}$ University Libraries

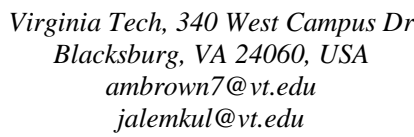

Received (Day Month Year)

Revised (Day Month Year)

Accepted (Day Month Year)

Keywords: amyloid- $\beta$; islet amyloid polypeptide (IAPP); polarizable molecular dynamics simulations, Drude oscillator

\section{Abstract}

Amyloids are a subset of intrinsically disordered proteins (IDPs) that self-assemble into cross- $\beta$ oligomers and fibrils. The structural plasticity of amyloids leads to sampling of metastable, low-molecular-weight oligomers that contribute to cytotoxicity. Of interest are amyloid- $\beta$ (A $\beta$ ) and islet amyloid polypeptide (IAPP), which are involved in the pathology of Alzheimer's disease and Type 2 Diabetes Mellitus, respectively. In addition to forming homogenous oligomers and fibrils, these species have been found to cross-aggregate in heterogeneous structures. Biophysical properties, including electronic effects, that are unique or conserved between homogenous and heterogenous amyloids oligomers are thus far unexplored. Here, we simulated homogenous and heterogenous amyloid oligomers of $\mathrm{A} \beta_{16-22}$ and $\mathrm{IAPP}_{20-29}$ fragments using the Drude oscillator model to investigate the impact of electronic polarization on the structural morphology and stability of preformed hexamers. Upon simulation of preformed, $\beta$-strand rich oligomers with Drude, structural rearrangement occurred causing some loss of $\beta$-strand structure in favor of random coil content for all oligomers. Homogenous $\mathrm{A} \beta_{16-22}$ was the most stable system, deriving stability from low polarization in hydrophobic residues and through salt bridge formation. Changes in polarization were observed primarily for $\mathrm{A} \beta_{16-22}$ residues in heterogenous cross-amyloid systems, displaying a decrease in charged residue dipole moments and an increase in hydrophobic sidechain dipole moments. This work is the first study utilizing the Drude-2019 force field with amyloid oligomers, providing insight into the impact of electronic effects on oligomer structure and highlighting the importance of different microenvironments on amyloid oligomer stability. 


\section{Introduction}

Intrinsically disordered proteins (IDPs) are involved in a variety of complex biological processes including cellular growth, protein modification, signaling cascades, and gene expression despite their lack of defined secondary structure ${ }^{l-5}$. Amyloids are an interesting subset of IDPs that self-assemble to form cross- $\beta$ aggregate fibrils. Some amyloids can misfold to form complex, cytotoxic oligomeric structures, which are associated with diseases including Alzheimer's disease (AD), Parkinson's disease, and Type 2 Diabetes Mellitus $(\mathrm{T} 2 \mathrm{DM})^{6-8}$. Amyloid aggregation is typically investigated via NMR spectroscopy $^{9}$, X-ray crystallography ${ }^{3}$, Förster resonance energy transfer (FRET) spectroscopy ${ }^{3,10}$, and more, but these techniques are limited in resolution due to the heterogeneity of amyloid conformational ensembles and the rate at which they aggregate and precipitate. Exploring how amyloids misfold and organize into low-molecular-weight oligomeric structures at the atomistic level is necessary for understanding the aggregation pathway of amyloids and the associated impact on disease progression.

Two commonly studied and disease-related amyloids are amyloid- $\beta$ (A $\beta)$ and islet amyloid polypeptide (IAPP). A $\beta$ is involved in the disease progression of AD whereas IAPP is co-secreted with insulin in the pancreas and has been associated with loss of pancreatic $\beta$-cell mass in T2DM. Typically, A $\beta$ and IAPP exacerbate these disease states by self-assembling and forming homogenous oligomeric intermediates inducing cell death, exerting cytotoxicity via ion leakage and perturbing membrane integrity ${ }^{11-13}$. These peptides then rearrange into a highly organized, $\beta$-sheet rich fibrillar morphologies that are less cytotoxic than low-molecular-weight oligomeric species ${ }^{14-16}$. To further compound the complexity of amyloid folding mechanisms and associated cytotoxicity, IAPP can potentiate $\mathrm{AD}$ via cross-amyloid interactions with $\mathrm{A} \beta$, or cross-seeding, forming heterogenous aggregates ${ }^{17}$.

Both $A \beta$ and IAPP display biochemical similarities that play a pivotal role in amyloid aggregation. Structurally, A $\beta$ and IAPP are short peptides that contain hydrophobic cores, contained within residues 16-22 of $\mathrm{A}^{18}$ and residues 20-29 of IAPP ${ }^{19,20}$ that are often used as experimental model systems for fibril growth. Homogenous fibril structures of these amyloids have been identified as organized steric zippers of monomeric $\beta$-strand repeats ${ }^{20}$, ${ }^{21}$. The cytotoxic oligomer intermediates form $\beta$-barrels, constructed from a ' $\beta$-strand- $\beta$ strand' motif for $\mathrm{A} \beta^{22,23}$ and IAPP ${ }^{24}$. Recent work has resolved the full-length and fragment fibril structures of $\mathrm{A} \beta^{25}$ and $\mathrm{IAPP}^{26}$, but there are limited data on the more cytotoxic lowmolecular-weight oligomeric intermediates of these peptides. Molecular dynamics (MD) simulations have been utilized to explore the formation of the amyloid oligomeric states given studying these amyloid oligomers is experimentally challenging.

Simulating the structures of $A \beta$ and IAPP and exploring their organization and formation of oligomer intermediates has been challenging due to force field inaccuracies, limitations on conformational sampling associated with limits in simulation lengths, and protein concentrations ${ }^{27}$. Many MD simulation force fields were designed for structured proteins and have shown to have limited accuracy and inadequate reproduction of 
experimentally characterized conformational ensembles due to overstabilization or overcompacting of IDP systems ${ }^{2}$. Employing MD simulations with force fields more robust for amyloid fragment simulation is necessary for accurately exploring amyloid aggregation pathways. Some success has been found utilizing Amber99SB ${ }^{28,} 29$, OPLS-AA ${ }^{30}$, GROMOS96 53A6 $6^{31}$, and GROMOS96 54A $7^{32}$, but lingering issues remain related to the overstabilization of certain types of secondary structures ${ }^{33}$. The need to utilize more robust force fields that can more accurately simulate and model amyloids such as A $\beta$ and IAPP is an essential next step for understanding disease pathology. Recent advancements in force field development that explicitly represent electronic polarization have allowed for more accurate representation of electrostatic effects, which may provide additional insight into the oligomeric organization of amyloids. Polarizable force fields may be more suitable for simulating these structures given their ability to explicitly represent dipole response as a function of local electric fields, an important feature when considering heterogeneous microenvironments that are found in oligomers and fibrils of $A \beta$ and IAPP.

Most common force fields for biomolecular systems employ a fixed-charged electrostatic model, limiting their ability to accurately represent anisotropic charge distribution and electronic interactions ${ }^{34,}{ }^{35}$. Polarizable force fields overcome this limitation by explicitly representing electronic degrees of freedom in a simulation system ${ }^{36}$. Two polarizable protein force fields that have recently emerged are $\mathrm{AMOEBA}^{37}$ and the classical Drude oscillator force field ${ }^{38-40}$. The Drude force field is based on the classical Drude oscillator model, in which auxiliary particles carrying negative charge are attached to non-hydrogen atoms, thereby modeling electronic degrees of freedom. The harmonic oscillations between the core atoms and their associated Drude particles model dipole response in the system. The Drude force field has been previously used to simulate A $\beta$ unfolding to reveal cooperative effects in stabilizing a key $\alpha$-helix in the central hydrophobic cluster ${ }^{41}$, and stabilizing quaternary interactions in amyloid fibrils ${ }^{42}$. Interestingly, previous simulations of amyloid fibrils with the Drude force field suggest that glycine electronic plasticity is particularly important in stabilizing complex fibril structures of multiple amyloid types ${ }^{42}$. To date, no studies on cytotoxic amyloid oligomer intermediates employing the Drude force field (or any polarizable force field) have been performed. Polarizable simulations may provide more detailed atomic insight into homogenous and heterogenous oligomer intermediates. This work seeks to explore the influence of electronic polarization on homogenous and heterogenous $\mathrm{A} \beta_{16-22}$ and IAPP 20 29 hexamer structures from previous simulations to better characterize the properties of these species. 


\section{Methods}

\subsection{System construction}

The starting coordinates for each system were extracted as the dominant cluster from RMSD clustering over the last $500 \mathrm{~ns}$ of previous simulations ${ }^{43}$ with the united-atom GROMOS96 53A6 force field (Figures S1-S3). Briefly, six peptide fragments were initially separated by at least $1.5 \mathrm{~nm}$, and aggregation was simulated over $2000 \mathrm{~ns}$. A total of 12 systems were simulated (4 replicates each of $\mathrm{A}_{16-22}, \mathrm{IAPP}_{20-29}$, and an equimolar mixture of $A \beta_{16-22}$ and $\mathrm{IAPP}_{20-29}$ ). We converted these united-atom structures into all-atom coordinates using the internal coordinate builder in $\mathrm{CHARMM}^{44}$, to be compatible with the CHARMM36m $(\mathrm{C} 36 \mathrm{~m})^{45}$ force field. The $\mathrm{N}$ - and C-termini of each peptide were capped with acetyl and amide groups, respectively, to avoid spurious end effects. Each system was solvated in a cubic box of CHARMM-modified TIP3P $\mathrm{P}^{46-48}$ water and $150 \mathrm{mM} \mathrm{KCl}$. Details of each system, including the number and type of atoms, are given in Table S1. Systems were energy-minimized in $\mathrm{CHARMM}^{44}$ for 500 steps of steepest descent minimization and 500 steps of adopted-basis Newton-Raphson (ABNR) minimization.

\subsection{Molecular Dynamics Simulations}

Following minimization, equilibration was performed for $1 \mathrm{~ns}$ in NAMD ${ }^{49}$. Position restraints $\left(5 \mathrm{kcal} \mathrm{mol}^{-1} \AA^{-2}\right)$ were applied to all non-hydrogen protein atoms and an NPT ensemble was maintained by regulating the temperature at $298 \mathrm{~K}$ with the Langevin thermostat method ${ }^{50,51}$ with a friction coefficient, $\gamma$, of $5 \mathrm{ps}^{-1}$. Pressure was set to $1 \mathrm{~atm}$ using the Langevin piston method $^{50}$; the oscillation period was $200 \mathrm{fs}$ and decay time was $100 \mathrm{fs}$. Periodic boundary conditions were applied in all directions and the short-range van der Waals forces were smoothly switched from zero to 10 to $12 \AA$. The particle mesh Ewald (PME) method $^{52,53}$ was used to calculate the electrostatic interactions with a real-space cutoff of $12 \AA$ and grid spacing of approximately $1 \AA$. Bonds to hydrogens atoms were held rigid using the $\mathrm{SHAKE}^{54}$ algorithm, allowing for the integration time step of $2 \mathrm{fs}$ in C36m simulations.

Following C36m equilibration, the systems were converted to the Drude-2019 polarizable mode ${ }^{39}$ in CHARMM by adding Drude oscillators and lone pairs to the equilibrated $\mathrm{C} 36 \mathrm{~m}$ coordinates. The TIP3P water model was converted to the polarizable SWM4-NDP ${ }^{55}$. The Drude oscillators were relaxed using 1000 steps of steepest descent and 500 steps of ABNR energy minimization in CHARMM. During this minimization, all real atoms were restrained with a force constant of $10^{8} \mathrm{kcal} \mathrm{mol}^{-1} \AA^{-2}$.

Equilibration of the polarizable systems was carried out for $1 \mathrm{~ns}$ in NAMD. The same restraints and constraints were applied, and the nonbonded settings were the same with the exception that the van der Waals potential, not force, was switched to zero from 10 to 12 $\AA$, as is the convention for the Drude-2019 force field. Drude simulations employed a dual 
Langevin thermostat to regulate temperature, in which real atoms were coupled to a thermostat at $298 \mathrm{~K}$ with $\gamma=5 \mathrm{ps}^{-1}$ and Drude oscillators coupled to a relative thermostat at $1 \mathrm{~K}$ and $\gamma=20 \mathrm{ps}^{-1}$. Following equilibration, unrestrained production simulations were performed with an in-house version ${ }^{56}$ of OpenMM ${ }^{57,58}$ version 7.1, using NVIDIA P100 GPUs. Four replicate simulations of each system were performed for $1 \mu \mathrm{s}$ each, for a total of $4 \mu$ s of total sampling for the $\mathrm{A} \beta_{16-22}, \mathrm{IAPP}_{20-29}$, and a heterogenous $\mathrm{A} \beta_{16-22} / \mathrm{IAPP}_{20-29}$ hexamers. The NPT ensemble was maintained using the same thermostat as during equilibration, however pressure was regulated at 1 atm using the Monte Carlo barostat in OpenMM with box scaling attempted every 25 integration steps.

\subsection{Analysis}

Secondary structure analysis was calculated according to the DSSP ${ }^{59}$ method as implemented in GROMACS ${ }^{60}$. Minimum distance calculations were performed as implemented in GROMACS. Standard CHARMM analysis tools were used to calculate radius of gyration $\left(\mathrm{R}_{\mathrm{g}}\right)$, root-mean-square deviation (RMSD), dipole moments, and solventaccessible surface area (SASA). Example CHARMM input scripts are available via the Open Science Framework at https://osf.io/mqcfd/.

\section{Results and Discussion}

Given the involvement of A $\beta$ and IAPP in AD and T2DM, extensive computational work has been performed studying amyloid oligomers, comprised of both full-length peptides $^{61-64}$ and fragments ${ }^{43,65,66}$, with additive force fields. The fixed point-charge approximation in these force fields fails to account for inevitable changes in local electric fields over the simulation trajectory ${ }^{40}$. As such, characterization of biomolecules using additive force fields may be insufficient to accurately describe structural changes and the evolution of associated heterogenous microenvironments that may depend on electronic polarization. Oligomers of $\mathrm{A}_{16-22}$ and $\mathrm{IAPP}_{20-29}$ have yet to be characterized in terms of these electronic effects. This work seeks to assess the structures adopted by amyloid oligomer fragments utilizing the Drude polarizable model and probe the role of polarization on oligomer compactness and structural changes.

\subsection{Polarizable Force Field Favors Random Coil Structures in Preformed Amyloid Oligomers}

It has been experimentally determined using methodologies such as transmission electron microscopy, that $A \beta$ oligomers form structures that are rich in antiparallel $\beta$ strands ${ }^{67}$ and can form $\beta$-barrels ${ }^{68}$. IAPP oligomers, in contrast, are thought to be more disordered $^{69}$, with residues 23-27 adopting transient $\beta$-strand structure on pathway to aggregation and fibril formation ${ }^{19}$. Very little experimental structural information is available for heterogenous $\mathrm{A} \beta / \mathrm{IAPP}$ oligomers; however, previous computational work has 
predicted the formation of pore-like cross-aggregate oligomers ${ }^{43}$. High $\beta$-sheet content in the hydrophobic amyloid fragments is often reported in computational work with both fulllength ${ }^{61,68}$ and fragment oligomer systems ${ }^{27,43,65}$; however, commonly used force fields such as GROMOS96 53A6 in SPC water ${ }^{70}$ and CHARMM22 $2^{71}$ in TIP3P water ${ }^{46}$ are thought to over-stabilize $\beta$-sheets and $\alpha$-helices, respectively, when simulating an $\mathrm{A} \beta_{40}$ monomer ${ }^{33}$. However, it should be noted that while GROMOS96 53A6 tends to overstabilize $\beta$-sheets, it was superior in replicating NMR data for an $\mathrm{A} \beta_{40}$ monomer in a force field comparison study ${ }^{72}$. The recently released Drude-2019 force field was optimized to address issues regarding under-stabilization of $\beta$-hairpins previously observed in the Drude-2013 force field ${ }^{39}$. However, the relative stability of secondary structures using this force field has not been explored in the context of amyloid quaternary structures, which are expected to be highly dynamic and heterogeneous.

The starting structures used in this work were rich in $\beta$-sheets, with replicates of $\mathrm{A} \beta_{16-22}$ and heterogeneous systems adopting pore-like structures (Figures S1-S3). At the outset of simulation, the oligomers contained $\sim 50 \% \beta$-sheet and $\sim 50 \%$ coil, whereas IAPP $_{20-29}$ was on average $\sim 60 \%$ coil and $\sim 40 \% \beta$-sheet (Table 1 ). Over the course of the 1 $\mu$ s simulations with the Drude force field, the $\beta$-strand content of the oligomers decreased, ultimately favoring random coil structures (Figure 1, Figures S4-S9, Table 1). $\beta$-strand structure was preserved primarily in hydrophobic regions of homogenous systems (Figure S10). In $\mathrm{A} \beta_{16-22}$ systems, residues Val18, Phe19, Phe20, and Ala21 retained $\beta$-strand as the dominant structure (Figure S10). Similarly, $\beta$-strand was dominant for residues Phe23, Gly24, Ala25, and Ile26 in IAPP ${ }_{20-29}$ systems (Figure S10). As such, our polarizable simulations of $A \beta_{16-22}$ and $\mathrm{IAPP}_{20-29}$ oligomers resulted in the peptides behaving more like intrinsically disordered peptides, favoring disorder, and sampling transient secondary structure. We hypothesize that this change in structure and modest rearrangement of structures is a result of adjusting for the now-present electronic effects as provided by the Drude force field. Interestingly, experimental evidence suggests that these oligomers, particularly $A \beta_{16-22}$, should adopt higher $\beta$-strand structure than we observed here ${ }^{68}$. In the case of $A \beta$ simulations and experimental results, full-length oligomerization is predicted to enhance formation of $\beta$-barrels, with residues 10-21 forming $\beta$-sheets at high probabilities $^{68}$. One possible explanation for this observation is a difference in force fields; that is, whereas the GROMOS96 53A6 simulations led to ordered structures rich in $\beta$ strands, the disordering observed in the Drude simulations suggests that this starting point was disfavored when modeled with explicit polarization. Another possibility is that $\beta$ strand structure is somewhat under-sampled when using the Drude-2019 force field, although there is no demonstration of such instability in ordered proteins against which the force field was validated ${ }^{39}$. Future studies of monomeric IDPs and other amyloid proteins should be pursued to determine whether $\beta$-strand content is consistent with experimental observations.

$\beta$-strand content was observed across all residues in the heterogeneous $A \beta_{16}$ ${ }_{22} / \mathrm{IAPP}_{20-29}$ oligomers (Figure S10). Hydrophobic residues in the central positions of each peptide were again the most enriched in these structures. It is notable that in the 
heterogeneous oligomers, $\beta$-strand content in $A \beta_{16-22}$ was slightly lower than in the homogeneous $A \beta_{16-22}$ oligomers, and $\beta$-strand content in IAPP $_{20-29}$ was comparable in both systems. The stability of $\beta$-strands in the heterogeneous oligomers suggests that the Drude2019 force field does not inherently underestimate the stability of $\beta$-strands, but rather is additionally influenced by microenvironments.
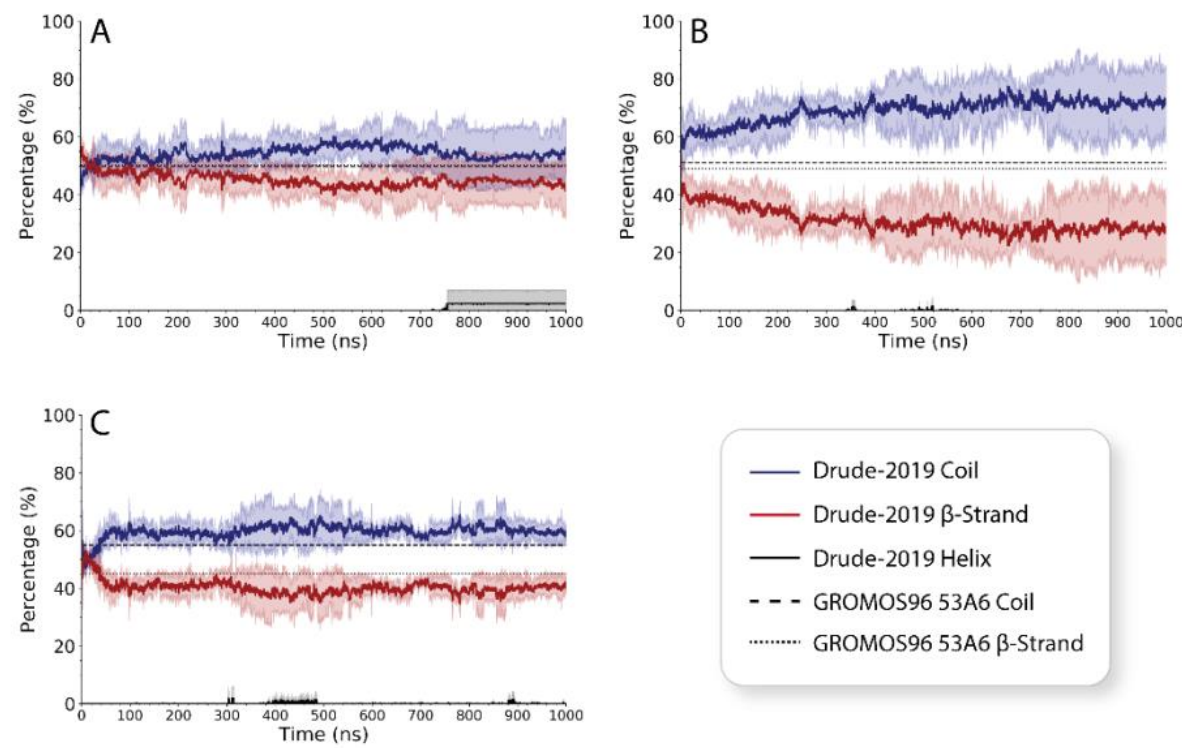

Figure 1. Average secondary structure percentage over time compared with the average $\beta$-strand and random coil percentages over the last $500 \mathrm{~ns}$ of simulation with GROMOS96 53A6 force field. Secondary structure percentage is shown for $\left.\mathbf{A}) \mathrm{A} \beta_{16-22}, \mathbf{B}\right) \mathrm{IAPP}_{20-29}$, and $\mathbf{C}$ ) $\mathrm{A} \beta_{16-22} / \mathrm{IAPP}_{20-29}$. Percentages for $\alpha$-helix (black), $\beta$-strand (red), and coil (blue) were averaged over all replicates. The standard deviation across replicates is shown as shading for each time series. The average $\alpha$-helical content over the last $500 \mathrm{~ns}$ of simulation with GROMOS96 53A6 is not shown, as it is near 0 . 
Table 1. Average secondary structure percentages for $\mathrm{A} \beta_{16-22}, \mathrm{IAPP}_{20-29}$, and $\mathrm{A} \beta_{16-22} / \mathrm{IAPP}_{(20-29}$, at simulation start, and over the last $500 \mathrm{~ns}$ of simulation duration. Averages taken across four replicates per system.

\begin{tabular}{|c|c|c|c|c|c|c|}
\hline \multirow[b]{2}{*}{ System } & \multicolumn{3}{|c|}{ Simulation Start $(\mathrm{t}=0)$} & \multicolumn{3}{|c|}{ Last $500 \mathrm{~ns}$} \\
\hline & Coil (\%) & $\beta$-Sheet (\%) & Helix (\%) & Coil (\%) & $\beta$-Sheet (\%) & Helix (\%) \\
\hline$A \beta_{16-22}$ & $43 \pm 3$ & $57 \pm 3$ & $0 \pm 0$ & $55 \pm 7$ & $44 \pm 6$ & $1 \pm 2$ \\
\hline IAPP $_{20-29}$ & $53 \pm 7$ & $47 \pm 7$ & $0 \pm 0$ & $72 \pm 9$ & $28 \pm 9$ & $0.01 \pm 0.03$ \\
\hline $\begin{array}{c}\mathbf{A} \boldsymbol{\beta}_{16-22} / \\
\mathbf{I A P P}_{20-29}\end{array}$ & $46 \pm 6$ & $54 \pm 6$ & $0 \pm 0$ & $60 \pm 3$ & $40 \pm 3$ & $0.1 \pm 0.1$ \\
\hline
\end{tabular}

In addition to increased coil content, low levels of $\alpha$-helical structure were sampled in at least one replicate of each system (Figures 1-2 and Figures S4-S9). This behavior was particularly prevalent in peptides that partially dissociated from the core oligomeric structure. Replicates 3 and 4 of $\mathrm{IAPP}_{20-29}$ in both homogenous and heterogeneous systems adopted transient helical structure; helices in homogenous $\mathrm{IAPP}_{20-29}$ were observed in residues Phe23, Gly24, and Ala25 (Figure 2A), whereas in heterogenous systems, helices were comprised of IAPP $20-29$ residues Ile26, Leu27, and Ser28 (Figure 2B). These helices were transient, with the most stable and long-lasting helices being sampled in replicate 3 of the heterogeneous $\mathrm{A} \beta_{16-22} / \mathrm{IAPP}_{20-29}$ system, persisting for $\sim 100 \mathrm{~ns}$ (Figures S8 and S9). In contrast, $A \beta_{16-22}$ replicate 4 adopted a stable helix comprised of residues Val18, Phe19, Phe20, and Ala21, which persisted over the last 250 ns of simulation (Figures 1, 2C, S4, S7). Interestingly, $\alpha$-helical structures were not sampled in measurable quantities over the course of simulations with GROMOS96 53A $6^{43}$, though this force field is known to understabilize such structures. The low levels of $\alpha$-helical structure in all systems simulated with Drude-2019 suggest that the polarizable force field models at least some of these peptides as sampling structures closer to a helix-coil equilibrium. 


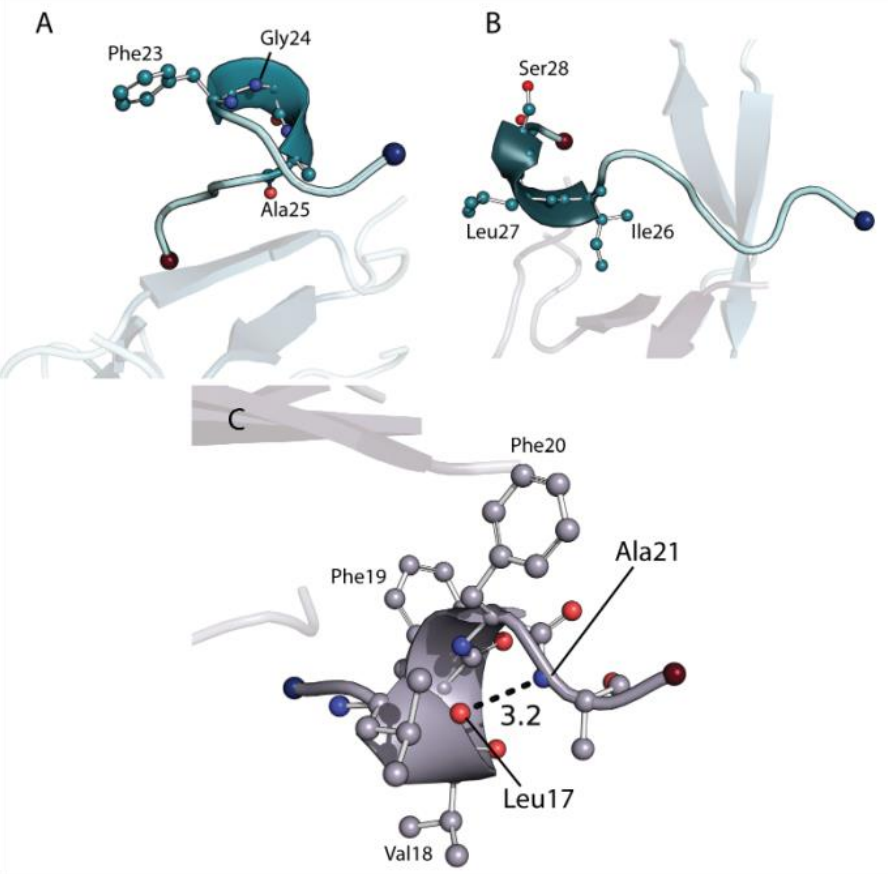

Figure 2. Presence of helical structure in amyloid peptides during simulation with the Drude-2019 force field. Helices adopted by $\mathbf{A}$ ) homogenous IAPP ${ }_{20-29}, \mathbf{B}$ ) heterogeneous IAPP $20-29$, and $\mathbf{C}$ ) homogenous $\mathrm{A} \beta_{16-22}$. Residues adopting helical configurations are shown as ball-and-stick, with carbons colored teal in $\mathrm{IAPP}_{20-29}$, and as light purple for $\mathrm{AB}_{16-22}$. Ball-and-stick nitrogen and oxygen atoms are colored blue and red, respectively. Peptides are shown as cartoon. N- and C-termini are shown as spheres, and colored dark blue and dark red, respectively. C) Hydrogen bond stabilizing $\alpha$-helix between Leu17 and Ala21 backbone is shown with distance shown in $\AA$.

\subsection{Stability of Homogenous Amyloid Oligomer Structures}

We sought to compare the simulated homogenous oligomers in terms of structural variation observed over time. To probe structural stability, we calculated radius of gyration $\left(\mathrm{R}_{\mathrm{g}}\right)$ and RMSD over the course of the trajectory (Table S2, Figures S11-S14). A $\beta_{16-22}$ oligomers were the most compact of all systems, with an average $R_{g}$ of $10.7 \pm 0.3 \AA$, and deviated the least from its starting structures, with an average RMSD of $1.3 \pm 0.1 \AA$ (Table S2). Hydrophobic packing is a critical component in amyloid oligomerization, and burial of hydrophobic residues should result in a depolarization of their sidechains as a function of being occluded from the aqueous solvent. Moreover, the structures of the different oligomers may result in aggregate-specific shifts in dipole moments. To investigate the association between packing and electronic polarization, sidechain dipole distributions 
were calculated for hydrophobic residues in both homogenous systems. Of the residues that are present in both $\mathrm{A} \beta_{16-22}$ and $\mathrm{IAPP}_{20-29}$ (Leu, Phe, and Ala), no change in sidechain dipole moment was observed between Leu residues (Figure 3A, Table 2). Sidechain dipole moment for Ala21 in $A \beta_{16-22}$ was marginally decreased compared to Ala25 in IAPP $20-29$ in terms of distribution (Figure 3D), although on average, there was no change (Table 2). The greatest differences in sidechain dipole moment were observed for Phe residues (Figure 3B, 3C, Table 2). On average, the sidechain dipole moment for Phe23 of homogenous $\mathrm{IAPP}_{20-29}(0.9 \pm 0.2 \mathrm{D})$ was elevated compared to homogenous $\mathrm{A} \beta_{16-22}$ residues Phe $19(0.8$ $\pm 0.1 \mathrm{D})$ and Phe20 $(0.7 \pm 0.1 \mathrm{D})$. The increased polarization of $\mathrm{IAPP}_{20-29}$ Phe23 relative to $A \beta$ Phe19 and Phe20 may influence aromatic contacts; homogenous $A \beta$, on average, had closer contacts between Phe residues $(7.0 \pm 0.3 \AA)$ than $\operatorname{IAPP}_{20-29}(8.0 \pm 3.0 \AA)$, as well as closer center-of-mass (COM) distances, on average, between Phe residues $(13.0 \pm 0.4 \AA$ vs. $17.0 \pm 5.0 \AA$ ) (Figure S15). Interestingly, Phe23 in homogenous $\mathrm{IAPP}_{20-29}$ was more solvent-exposed (194 $\pm 4 \AA^{2}$ solvent-accessible surface area) than Phe residues in homogenous $A \beta_{16-22}\left(187 \pm 4 \AA^{2}\right.$ and $178 \pm 3 \AA^{2}$ ) (Table S2 and S3, respectively). Together, these data suggest that increased solvent accessibility is consistent with higher sidechain dipole moments in Phe, and reflects the plasticity of the electronic structure of this residue. Phe residues in $A \beta_{16-22}$ may be driven inward to a greater extent since there are two of them, which is subsequently reflected in their depolarization as a function of solvent occlusion.

Table 2. Average sidechain dipole moments for common residues in homogenous $A \beta_{16-22}$ and homogenous

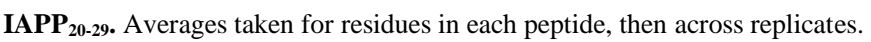

\begin{tabular}{|c|c|c|c|c|}
\hline \multirow{2}{*}{ A $_{\text {16-22 }}$ Residue } & IAPP $_{\text {20-29 }}$ Residue & $\begin{array}{c}\text { A } \boldsymbol{\beta}_{16-22} \text { Average } \\
\text { Sidechain Dipole } \\
\text { Moment (D) }\end{array}$ & $\begin{array}{c}\text { IAPP } \\
\text { Sidechain Dipole } \\
\text { Moment (D) }\end{array}$ & Difference \\
\hline Leu17 & Leu27 & $0.7 \pm 0.1$ & $0.7 \pm 0.1$ & 0.0 \\
\hline Phe19 & Phe23 & $0.8 \pm 0.1$ & $0.9 \pm 0.2$ & 0.1 \\
\hline Phe20 & Phe23 & $0.7 \pm 0.1$ & $0.9 \pm 0.2$ & 0.0 \\
\hline Ala21 & Ala25 & $0.7 \pm 0.04$ & $0.7 \pm 0.05$ & 0.0 \\
\hline
\end{tabular}

$\mathrm{A} \beta_{16-22}$ contains charged residues Lys 16 and Glu22 flanking the hydrophobic core. We hypothesized that the formation of salt bridges may influence the increased relative stability seen in homogenous $A \beta_{16-22}$ systems. In replicates 2-4, $A \beta_{16-22}$ Lys 16 and Glu22 residues engaged in salt bridges in $\sim 30 \%$ of frames across the trajectory (Table S5). Salt bridge formation was elevated in replicate 1, with charged residues engaging in salt bridges in 56\% of frames (Figure 3E and 3F). Replicate 1 of homogeneous $A \beta_{16-22}$ was the most stable in terms of RMSD and $R_{g}$, although these differences may not be significant (Table S6). Furthermore, replicate 1 of homogenous $A \beta_{16-22}$ had the highest percentage of $\beta$-strand 
structure over the course of simulation, and was subject to less variation in secondary structure (Table S6). These observations indicate that salt bridge formation in homogenous $\mathrm{A} \beta_{16-22}$ play an important role in modulating oligomer stability. Many nonpolarizable force fields overestimate the stability of salt bridges, and it has previously been proposed that implicitly polarized or explicitly polarizable force fields may be more suitable models for such interactions ${ }^{73}$. Our results here suggest that modeling salt bridges correctly has implications for amyloid oligomer stability and should be investigated more thoroughly in the future.
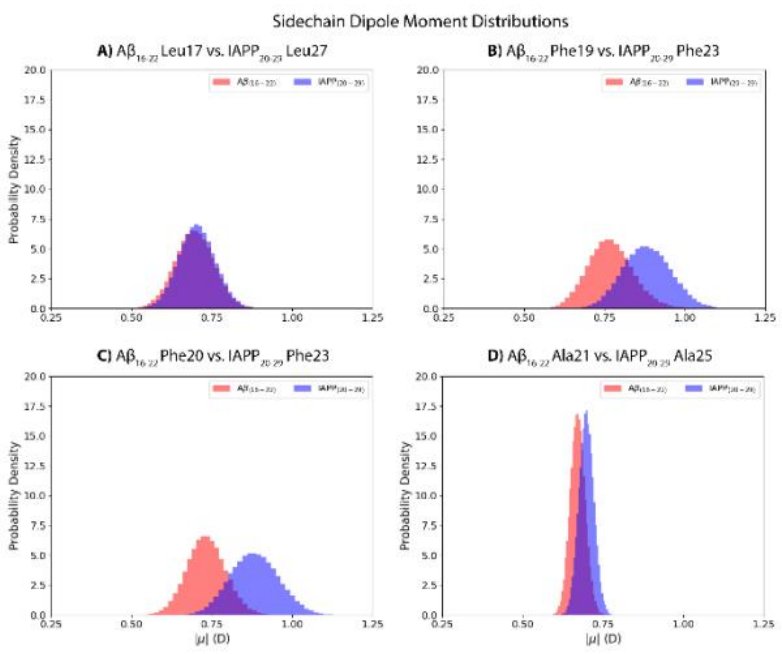

E

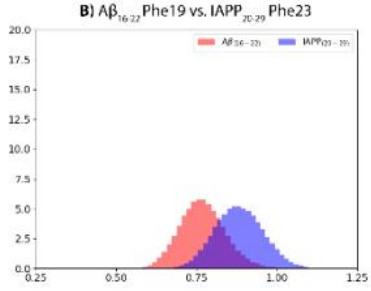

25

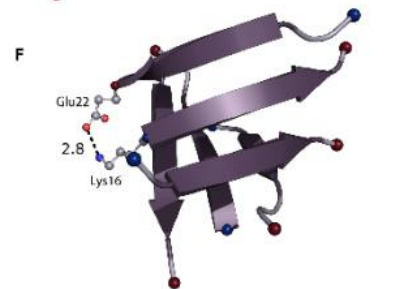

Figure 3. Sidechain dipole moment distributions in $\mathrm{A} \beta_{16-22}$ and $\mathrm{IAPP}_{20-29}$ systems and salt bridge formation. AD) Sidechain dipole moment distributions for common residues in $\mathrm{A} \beta_{16-22}$ and $\left.\mathrm{IAPP}_{20-29}, \mathbf{A}\right)$ Leu 17 and Leu27, B) Phe19 and Phe23, C) Phe20 and Phe24, and D) Ala21 and Ala25. E-F) Observed salt bridge between Glu22 and Lys16 of $A \beta_{16-22}$ system. Peptides shown as cartoon, with $\mathrm{N}$ - and $\mathrm{C}$ - termini shown as spheres, colored blue and red, respectively. Glu22 and Lys16 are shown as ball-and-stick, with oxygens and nitrogen atoms colored red and blue, respectively. The distance measurement is shown in $\AA$.

\subsection{Impact of Dipole Moments on Homogenous and Heterogenous Amyloid Oligomers}

Heterogenous $\mathrm{A} \beta_{16-22} / \mathrm{IAPP}_{20-29}$ oligomers differed from their homogenous counterparts in terms of stability and compactness. The heterogeneous $\mathrm{A} \beta_{16-22} / \mathrm{IAPP}_{20-29}$ oligomers maintained $R_{g}$ values that were intermediate between homogeneous $A \beta_{16-22}$ and IAPP $_{20-29}$ oligomers (Table S2), reflecting the combination of their different sizes (A $\beta_{16-22}$ being smaller than $\mathrm{IAPP}_{20-29)}$. Calculations of $\mathrm{R}_{\mathrm{g}}$ over time also revealed that heterogeneous $\mathrm{A} \beta_{16-22} / \mathrm{IAPP}_{20-29}$ systems were less susceptible to having a peptide dissociate from the core oligomer structure than were the homogenous systems (Figure $\mathrm{S} 11$ ). To assess how differences in microenvironments may affect stability, we compared 
sidechain dipole moment distributions btabetween residues in the respective systems. Engagement in the heterogeneous $\mathrm{A} \beta_{16-22} / \mathrm{IAPP}_{20-29}$ oligomers led to a depolarization of $\mathrm{A} \beta_{16-22}$ charged residues Lys16 and Glu22 and increased polarization of hydrophobic sidechains of Leu17, Val18, and Phe20 (Figure 4A, Figure S16). The depolarization of Lys16 and Glu22 are reflected in the lack of salt bridge formation compared to homogenous $A \beta_{16-22}$ (Table S4). Previous simulations of mature amyloid fibrils with Drude indicate that sidechain dipole moments for charged residues increase when engaged in salt bridges $^{42}$, and the results obtained here suggest this phenomenon is true in low-molecularweight oligomers and therefore may be a persistent feature along the amyloid aggregation pathway.

The polarization of hydrophobic $A \beta_{16-22}$ residues Leu17, Val18, and Phe20 in heterogeneous systems may be influenced the introduction of polar residues from $\mathrm{IAPP}_{20}$ 29 to the microenvironment. These shifts in hydrophobic polarization may stabilize interesting configurations; distance matrices show that on average, Glu22 formed closer contacts with hydrophobic residue sidechains in heterogenous systems (Table S7). In replicate 4, Glu22 interacts with hydrophobic residues Leu17, Val18, and Phe20 of A $\beta$, and Ile26 of IAPP at the interface of solvent and the hydrophobic core (Figure 4B). Interestingly, Phe19 and Ala21 were resistant to shifts in polarization. In heterogeneous systems, Phe19 was less exposed to solvent $\left(116 \pm 5 \AA^{2}\right)$ than Phe20 $\left(129 \pm 6 \AA^{2}\right)$, suggesting this residue experienced more hydrophobic packing, and thus less influenced by polar residues (Table S3).

Few differences were observed between $\mathrm{IAPP}_{20-29}$ dipole moments in homogenous and heterogeneous systems, with the exception of Gly24 (Figures 4C, S17, and S18). Gly24 dipole moment differences between heterogeneous and homogenous systems did not follow a clear trend, suggesting that this residue is highly sensitive to its microenvironment (Figure S18). There are no strong correlations between Gly24 and SASA in heterogeneous systems (Figure S19). The lack of correlation to SASA suggests the residue microenvironment in heterogeneous systems has more influence over Gly24 dipole moments than solvent exposure. Our previous simulations of amyloid fibrils with the Drude force field suggested that glycine residues exhibit electronic plasticity in response to microenvironment changes to promote the stabilization of a given structure ${ }^{42}$. The relatively increased stability in heterogeneous oligomers with respect to $\mathrm{IAPP}_{20-29}$ may be attributed to glycine adjusting its electronic structure to favor stability. 

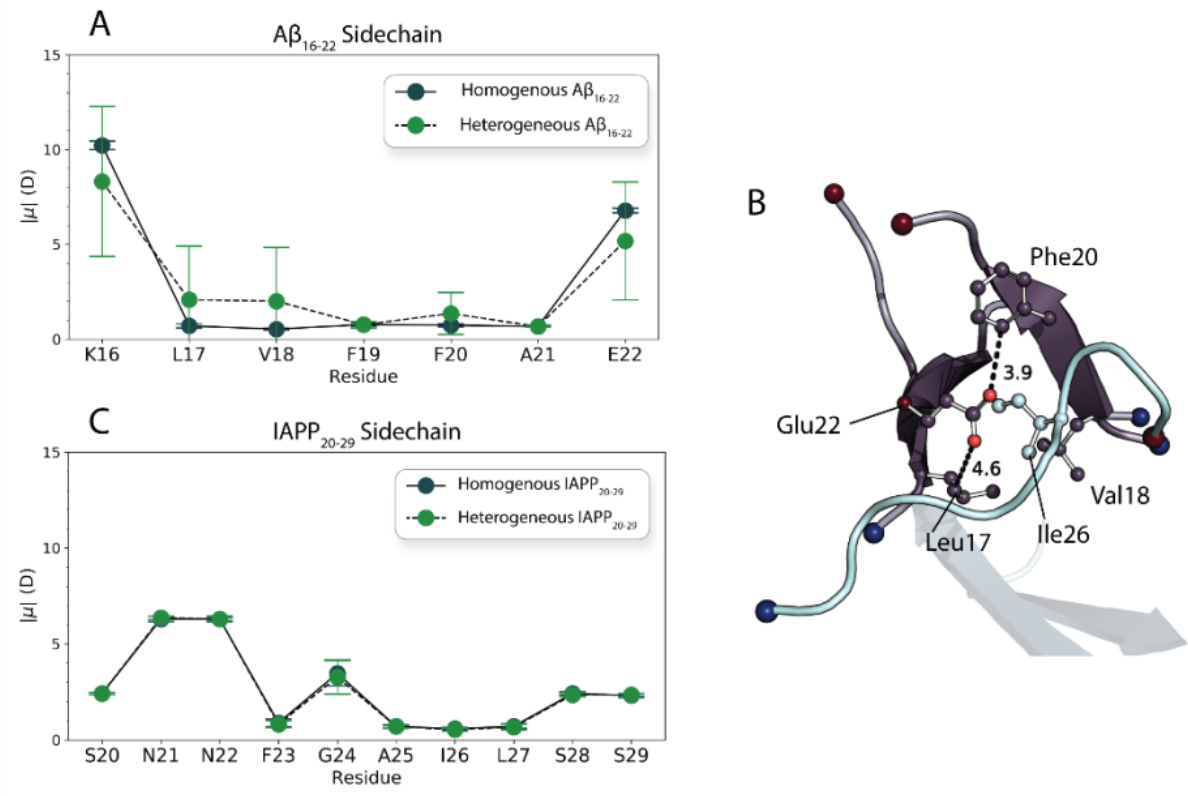

Figure 4. Sidechain dipole moment comparison and intermolecular interactions. A) Average sidechain dipole moments per residue in (dark blue) homogenous $A \beta_{16-22}$ and (green) heterogeneous $A \beta_{16-22}$. B) Glu22 shows more frequent contacts with hydrophobic residues in homogeneous systems. Image shows Glu22 interacting with Leu17, Val18, and Phe20 of $A \beta_{16-22}$, and Ile26 of IAPP $20-29$. A $\beta$ coils colored lavender, and $\beta$-sheets colored as dark purple. IAPP $20-29$ coil colored as light blue. Residues in the vicinity of Glu22 are shown as ball-and-stick, with carbons colored dark purple (A $\beta$ ) and light blue (IAPP). Oxygens in ball-and-stick are colored red. N-and C- termini are shown as spheres and colored blue and red, respectively. C) Average sidechain dipole moments per residue in (dark blue) homogeneous IAPP $_{20-29}$ and (green) heterogeneous IAPP $_{20-29}$. A and C) Averages are taken across all peptides and replicates over the entire trajectory.

\section{Conclusions}

This work sought to determine the impact of electronic polarization on homogenous and heterogenous amyloid oligomers. Atomistic detail of the structural morphologies of these metastable oligomers is mostly unknown, and computer simulations are sometimes limited in accuracy as a result of force field inaccuracies that lead to imbalance of different secondary structure elements. Utilizing the Drude-2019 force field, we observed that structural rearrangement occurred, most notably a modest decline in $\beta$-strand structure as compared to starting structures. The most stable system simulated was $A \beta_{16-22}$, whose saltbridge formation modulated its relatively lower $\mathrm{R}_{\mathrm{g}}$ and RMSD compared to other systems. Additionally, residues Phe19 and Phe20 in A $\beta_{16-22}$ had lower sidechain dipole moments and were less solvent accessible than Phe23 in $\mathrm{IAPP}_{20-29}$, which contributed to stronger hydrophobic packing and compactness. In heterogeneous systems, $\mathrm{A} \beta_{16-22}$ experienced a depolarization of charged residues Lys16 and Glu22, and a polarization of hydrophobic 
residues Leu17, Val18, and Phe20. These changes can be attributed to lack of salt bridge formation in heterogeneous systems and the introduction of polar residues from $\operatorname{IAPP}_{20-29}$. Very little difference was observed between homogenous and heterogeneous IAPP $20-29$ in terms of polarization, except for Gly24, whose electronic plasticity may work to stabilize heterogenous microenvironments. This work highlights the impact of electronic polarization on the stability and properties of amyloid oligomers, with special focus on the role of microenvironments in maintaining oligomer stability.

\section{Acknowledgments}

The authors thank Virginia Tech Advanced Research Computing for computing time and resources. This work was supported by the National Institutes of Health (grant R35GM133754, to JAL).

\section{References}

[1] Deiana, A., Forcelloni, S., Porrello, A., and Giansanti, A. (2019) Intrinsically disordered proteins and structured proteins with intrinsically disordered regions have different functional roles in the cell, PLoS One 14, e0217889.

[2] Wang, W. (2021) Recent advances in atomic molecular dynamics simulation of intrinsically disordered proteins, Physical Chemistry Chemical Physics 23, 777-784.

[3] Wang, A., Peng, X., Li, Y., Zhang, D., Zhang, Z., and Li, G. (2021) Quality of force fields and sampling methods in simulating pepX peptides: a case study for intrinsically disordered proteins, Physical Chemistry Chemical Physics 23, 2430-2437.

[4] van der Lee, R., Buljan, M., Lang, B., Weatheritt, R. J., Daughdrill, G. W., Dunker, A. K., Fuxreiter, M., Gough, J., Gsponer, J., Jones, D. T., Kim, P. M., Kriwacki, R. W., Oldfield, C. J., Pappu, R. V., Tompa, P., Uversky, V. N., Wright, P. E., and Babu, M. M. (2014) Classification of Intrinsically Disordered Regions and Proteins, Chemical Reviews 114, 6589-6631.

[5] Wright, P. E., and Dyson, H. J. (2015) Intrinsically disordered proteins in cellular signalling and regulation, Nature Reviews Molecular Cell Biology 16, 18-29.

[6] Nguyen, P. H., Ramamoorthy, A., Sahoo, B. R., Zheng, J., Faller, P., Straub, J. E., Dominguez, L., Shea, J.-E., Dokholyan, N. V., De Simone, A., Ma, B., Nussinov, R., Najafi, S., Ngo, S. T., Loquet, A., Chiricotto, M., Ganguly, P., McCarty, J., Li, M. S., Hall, C., Wang, Y., Miller, Y., Melchionna, S., Habenstein, B., Timr, S., Chen, J., Hnath, B., Strodel, B., Kayed, R., Lesné, S., Wei, G., Sterpone, F., Doig, A. J., and Derreumaux, P. (2021) Amyloid Oligomers: A Joint Experimental/Computational Perspective on Alzheimer's Disease, Parkinson's Disease, Type II Diabetes, and Amyotrophic Lateral Sclerosis, Chemical Reviews 121, 2545-2647. 
[7] Eisenberg, D., and Jucker, M. (2012) The amyloid state of proteins in human diseases, Cell 148, 1188-1203.

[8] Iadanza, M. G., Jackson, M. P., Hewitt, E. W., Ranson, N. A., and Radford, S. E. (2018) A new era for understanding amyloid structures and disease, Nat Rev Mol Cell Biol 19, 755-773.

[9] Samdin, T. D., Kreutzer, A. G., and Nowick, J. S. (2021) Exploring amyloid oligomers with peptide model systems, Current Opinion in Chemical Biology 64, 106-115.

[10] LeBlanc, S. J., Kulkarni, P., and Weninger, K. R. (2018) Single Molecule FRET: A Powerful Tool to Study Intrinsically Disordered Proteins, Biomolecules 8, 140.

[11] Berridge, M. J. (2010) Calcium hypothesis of Alzheimer's disease, Pflugers Arch 459, 441-449.

[12] Di Scala, C., Yahi, N., Boutemeur, S., Flores, A., Rodriguez, L., Chahinian, H., and Fantini, J. (2016) Common molecular mechanism of amyloid pore formation by Alzheimer's $\beta$ amyloid peptide and $\alpha$-synuclein, Scientific Reports 6, 28781.

[13] Kayed, R., Head, E., Thompson, J. L., McIntire, T. M., Milton, S. C., Cotman, C. W., and Glabe, C. G. (2003) Common structure of soluble amyloid oligomers implies common mechanism of pathogenesis, Science 300, 486-489.

[14] Siddiqi, M. K., Malik, S., Majid, N., Alam, P., and Khan, R. H. (2019) Cytotoxic species in amyloid-associated diseases: Oligomers or mature fibrils, Adv Protein Chem Struct Biol 118, 333-369.

[15] Breydo, L., Kurouski, D., Rasool, S., Milton, S., Wu, J. W., Uversky, V. N., Lednev, I. K., and Glabe, C. G. (2016) Structural differences between amyloid beta oligomers, Biochem Biophys Res Commun 477, 700-705.

[16] Breydo, L., and Uversky, V. N. (2015) Structural, morphological, and functional diversity of amyloid oligomers, FEBS letters 589, 2640-2648.

[17] Moreno-Gonzalez, I., Edwards Iii, G., Salvadores, N., Shahnawaz, M., Diaz-Espinoza, R., and Soto, C. (2017) Molecular interaction between type 2 diabetes and Alzheimer's disease through cross-seeding of protein misfolding, Mol Psychiatry 22, 1327-1334.

[18] Balbach, J. J., Ishii, Y., Antzutkin, O. N., Leapman, R. D., Rizzo, N. W., Dyda, F., Reed, J., and Tycko, R. (2000) Amyloid Fibril Formation by A $\beta 16-22$, a Seven-Residue Fragment of the Alzheimer's $\beta$-Amyloid Peptide, and Structural Characterization by Solid State NMR, Biochemistry 39, 13748-13759.

[19] Buchanan, L. E., Dunkelberger, E. B., Tran, H. Q., Cheng, P. N., Chiu, C. C., Cao, P., Raleigh, D. P., de Pablo, J. J., Nowick, J. S., and Zanni, M. T. (2013) Mechanism of IAPP amyloid fibril formation involves an intermediate with a transient beta-sheet, Proceedings of the National Academy of Sciences of the United States of America 110, 19285-19290.

[20] Sawaya, M. R., Sambashivan, S., Nelson, R., Ivanova, M. I., Sievers, S. A., Apostol, M. I., Thompson, M. J., Balbirnie, M., Wiltzius, J. J. W., McFarlane, H. T., Madsen, A. Ø., Riekel, C., and Eisenberg, D. (2007) Atomic structures of amyloid cross- $\beta$ spines reveal varied steric zippers, Nature 447, 453-457.

[21] Park, J., Kahng, B., and Hwang, W. (2009) Thermodynamic selection of steric zipper patterns in the amyloid cross-beta spine, PLoS Comput Biol 5, e1000492-e1000492.

[22] Jang, H., Arce, F. T., Ramachandran, S., Capone, R., Azimova, R., Kagan, B. L., Nussinov, R., and Lal, R. (2010) Truncated beta-amyloid peptide channels provide an alternative mechanism for Alzheimer's Disease and Down syndrome, Proceedings of the National Academy of Sciences of the United States of America 107, 6538-6543. 
[23] Jang, H., Zheng, J., and Nussinov, R. (2007) Models of beta-amyloid ion channels in the membrane suggest that channel formation in the bilayer is a dynamic process, Biophysical journal 93, 1938-1949.

[24] Mao, X.-B., Wang, C.-X., Wu, X.-K., Ma, X.-J., Liu, L., Zhang, L., Niu, L., Guo, Y.-Y., Li, D.H., Yang, Y.-L., and Wang, C. (2011) Beta structure motifs of islet amyloid polypeptides identified through surface-mediated assemblies, Proceedings of the National Academy of Sciences 108, 19605.

[25] Gremer, L., Schölzel, D., Schenk, C., Reinartz, E., Labahn, J., Ravelli, R. B. G., Tusche, M., Lopez-Iglesias, C., Hoyer, W., Heise, H., Willbold, D., and Schröder, G. F. (2017) Fibril structure of amyloid- $\beta(1-42)$ by cryo-electron microscopy, Science 358, 116-119.

[26] Gallardo, R., Iadanza, M. G., Xu, Y., Heath, G. R., Foster, R., Radford, S. E., and Ranson, N. A. (2020) Fibril structures of diabetes-related amylin variants reveal a basis for surfacetemplated assembly, Nat Struct Mol Biol 27, 1048-1056.

[27] Strodel, B. (2021) Amyloid aggregation simulations: challenges, advances and perspectives, Current Opinion in Structural Biology 67, 145-152.

[28] Hornak, V., Abel, R., Okur, A., Strockbine, B., Roitberg, A., and Simmerling, C. (2006) Comparison of multiple Amber force fields and development of improved protein backbone parameters, Proteins 65, 712-725.

[29] Wickstrom, L., Okur, A., and Simmerling, C. (2009) Evaluating the performance of the ff99SB force field based on NMR scalar coupling data, Biophys $J$ 97, 853-856.

[30] Kaminski, G. A., Friesner, R. A., Tirado-Rives, J., and Jorgensen, W. L. (2001) Evaluation and Reparametrization of the OPLS-AA Force Field for Proteins via Comparison with Accurate Quantum Chemical Calculations on Peptides, The Journal of Physical Chemistry B 105, 6474-6487.

[31] Oostenbrink, C., Villa, A., Mark, A. E., and van Gunsteren, W. F. (2004) A biomolecular force field based on the free enthalpy of hydration and solvation: the GROMOS force-field parameter sets 53A5 and 53A6, Journal of computational chemistry 25, 1656-1676.

[32] Schmid, N., Eichenberger, A. P., Choutko, A., Riniker, S., Winger, M., Mark, A. E., and van Gunsteren, W. F. (2011) Definition and testing of the GROMOS force-field versions 54A7 and 54B7, European Biophysics Journal 40, 843.

[33] Gerben, S. R., Lemkul, J. A., Brown, A. M., and Bevan, D. R. (2014) Comparing atomistic molecular mechanics force fields for a difficult target: a case study on the Alzheimer's amyloid $\beta$-peptide, J Biomol Struct Dyn 32, 1817-1832.

[34] Lemkul, J. A. (2021) Preparing and Analyzing Polarizable Molecular Dynamics Simulations with the Classical Drude Oscillator Model, Methods Mol Biol 2315, 219-240.

[35] Jing, Z., Liu, C., Cheng, S. Y., Qi, R., Walker, B. D., Piquemal, J. P., and Ren, P. (2019) Polarizable Force Fields for Biomolecular Simulations: Recent Advances and Applications, Annu Rev Biophys 48, 371-394.

[36] Lemkul, J. A. (2020) Chapter One - Pairwise-additive and polarizable atomistic force fields for molecular dynamics simulations of proteins, In Progress in Molecular Biology and Translational Science (Strodel, B., and Barz, B., Eds.), pp 1-71, Academic Press.

[37] Shi, Y., Xia, Z., Zhang, J., Best, R., Wu, C., Ponder, J. W., and Ren, P. (2013) Polarizable Atomic Multipole-Based AMOEBA Force Field for Proteins, Journal of Chemical Theory and Computation 9, 4046-4063. 
[38] Lopes, P. E. M., Huang, J., Shim, J., Luo, Y., Li, H., Roux, B., and MacKerell, A. D. (2013) Polarizable Force Field for Peptides and Proteins Based on the Classical Drude Oscillator, Journal of Chemical Theory and Computation 9, 5430-5449.

[39] Lin, F.-Y., Huang, J., Pandey, P., Rupakheti, C., Li, J., Roux, B. t., and MacKerell, A. D. (2020) Further Optimization and Validation of the Classical Drude Polarizable Protein Force Field, Journal of Chemical Theory and Computation 16, 3221-3239.

[40] Lemkul, J. A., Huang, J., Roux, B., and MacKerell, A. D. (2016) An Empirical Polarizable Force Field Based on the Classical Drude Oscillator Model: Development History and Recent Applications, Chemical Reviews 116, 4983-5013.

[41] Lemkul, J. A., Huang, J., and MacKerell, A. D., Jr. (2015) Induced Dipole-Dipole Interactions Influence the Unfolding Pathways of Wild-Type and Mutant Amyloid $\beta$-Peptides, J Phys Chem B 119, 15574-15582.

[42] Davidson, D. S., Brown, A. M., and Lemkul, J. A. (2018) Insights into Stabilizing Forces in Amyloid Fibrils of Differing Sizes from Polarizable Molecular Dynamics Simulations, Journal of Molecular Biology 430, 3819-3834.

[43] Cramer, N., Kawecki, G., King, K. M., Bevan, D. R., and Brown, A. M. (2021) Insight into Cross-Amyloid Interactions and Morphologies: Molecular Dynamics Simulations of Model Peptide Fragments of Amyloid- $\beta(\mathrm{A} \beta<\mathrm{sub}>16-22</ \mathrm{sub}>)$ and Islet Amyloid Polypeptide (IAPP<sub>20-29</sub>), bioRxiv, 2021.2009.2026.461861.

[44] Brooks, B. R., Brooks III, C. L., Mackerell Jr., A. D., Nilsson, L., Petrella, R. J., Roux, B., Won, Y., Archontis, G., Bartels, C., Boresch, S., Caflisch, A., Caves, L., Cui, Q., Dinner, A. R., Feig, M., Fischer, S., Gao, J., Hodoscek, M., Im, W., Kuczera, K., Lazaridis, T., Ma, J., Ovchinnikov, V., Paci, E., Pastor, R. W., Post, C. B., Pu, J. Z., Schaefer, M., Tidor, B., Venable, R. M., Woodcock, H. L., Wu, X., Yang, W., York, D. M., and Karplus, M. (2009) CHARMM: The biomolecular simulation program, Journal of computational chemistry 30, 1545-1614.

[45] Huang, J., Rauscher, S., Nawrocki, G., Ran, T., Feig, M., de Groot, B. L., Grubmüller, H., and MacKerell, A. D., Jr. (2017) CHARMM36m: an improved force field for folded and intrinsically disordered proteins, Nat Methods 14, 71-73.

[46] Jorgensen, W. L., Chandrasekhar, J., and Madura, J. D. (1983) Comparison of simple potential functions for simulating liquid water, The Journal of Chemical Physics 79.

[47] Neria, E., Fischer, S., and Karplus, M. (1996) Simulation of activation free energies in molecular systems, The Journal of Chemical Physics 105, 926-935.

[48] Durell, S. R., Brooks, B. R., and Ben-Naim, A. (1994) Solvent-Induced Forces between Two Hydrophilic Groups, The Journal of Physical Chemistry 98, 2198-2202.

[49] Jiang, W., Hardy, D. J., Phillips, J. C., MacKerell, A. D., Schulten, K., and Roux, B. (2011) High-Performance Scalable Molecular Dynamics Simulations of a Polarizable Force Field Based on Classical Drude Oscillators in NAMD, The Journal of Physical Chemistry Letters 2, 87-92.

[50] Feller, S. E., Zhang, Y., Pastor, R. W., and Brooks, B. R. (1995) Constant pressure molecular dynamics simulation: The Langevin piston method, The Journal of Chemical Physics 103, 4613-4621.

[51] Martyna, G. J., Tobias, D. J., and Klein, M. L. (1994) Constant pressure molecular dynamics algorithms, The Journal of Chemical Physics 101, 4177-4189. 
[52] Darden, T., York, D., and Pedersen, L. (1993) Particle Mesh Ewald: An Nlog (N) Method for Ewald Sums in Large Systems, Vol. 98.

[53] Essmann, U., Perera, L., Berkowitz, M. L., Darden, T., Lee, H., and Pedersen, L. G. (1995) A smooth particle mesh Ewald method, The Journal of Chemical Physics 103, 8577-8593.

[54] Ryckaert, J.-P., Ciccotti, G., and Berendsen, H. J. C. (1977) Numerical integration of the cartesian equations of motion of a system with constraints: molecular dynamics of nalkanes, Journal of Computational Physics 23, 327-341.

[55] Lamoureux, G., Harder, E., Vorobyov, I. V., Roux, B., and MacKerell, A. D. (2006) A polarizable model of water for molecular dynamics simulations of biomolecules, Chemical Physics Letters 418, 245-249.

[56] Huang, J., Lemkul, J. A., Eastman, P. K., and MacKerell Jr, A. D. (2018) Molecular dynamics simulations using the drude polarizable force field on GPUs with OpenMM: Implementation, validation, and benchmarks, Journal of computational chemistry 39, 1682-1689.

[57] Lamoureux, G., and Roux, B. (2003) Modeling induced polarization with classical Drude oscillators: Theory and molecular dynamics simulation algorithm, The Journal of Chemical Physics 119, 3025-3039.

[58] Eastman, P., Swails, J., Chodera, J. D., McGibbon, R. T., Zhao, Y., Beauchamp, K. A., Wang, L. P., Simmonett, A. C., Harrigan, M. P., Stern, C. D., Wiewiora, R. P., Brooks, B. R., and Pande, V. S. (2017) OpenMM 7: Rapid development of high performance algorithms for molecular dynamics, PLoS Comput Biol 13, e1005659.

[59] Kabsch, W., and Sander, C. (1983) Dictionary of protein secondary structure: pattern recognition of hydrogen-bonded and geometrical features, Biopolymers 22, 2577-2637.

[60] Abraham, M. J., Murtola, T., Schulz, R., Páll, S., Smith, J. C., Hess, B., and Lindahl, E. (2015) GROMACS: High performance molecular simulations through multi-level parallelism from laptops to supercomputers, SoftwareX 1-2, 19-25.

[61] Brown, Anne M., and Bevan, David R. (2016) Molecular Dynamics Simulations of Amyloid $\beta$ Peptide (1-42): Tetramer Formation and Membrane Interactions, Biophysical Journal 111, 937-949.

[62] Mehrazma, B., and Rauk, A. (2019) Exploring Amyloid- $\beta$ Dimer Structure Using Molecular Dynamics Simulations, The Journal of Physical Chemistry A 123, 4658-4670.

[63] Liu, N., Duan, M., and Yang, M. (2017) Structural Properties of Human IAPP Dimer in Membrane Environment Studied by All-Atom Molecular Dynamics Simulations, Scientific Reports 7, 7915.

[64] Moore, S. J., Sonar, K., Bharadwaj, P., Deplazes, E., and Mancera, R. L. (2018) Characterisation of the Structure and Oligomerisation of Islet Amyloid Polypeptides (IAPP): A Review of Molecular Dynamics Simulation Studies, Molecules 23, 2142.

[65] Illig, A.-M., and Strodel, B. (2020) Performance of Markov State Models and Transition Networks on Characterizing Amyloid Aggregation Pathways from MD Data, Journal of Chemical Theory and Computation 16, 7825-7839.

[66] Okumura, H., and Itoh, S. G. (2020) Molecular dynamics simulations of amyloid- $\beta(16-22)$ peptide aggregation at air-water interfaces, J Chem Phys 152, 095101.

[67] Cerf, E., Sarroukh, R., Tamamizu-Kato, S., Breydo, L., Derclaye, S., Dufrêne, Y. F., Narayanaswami, V., Goormaghtigh, E., Ruysschaert, J. M., and Raussens, V. (2009) 
Antiparallel beta-sheet: a signature structure of the oligomeric amyloid beta-peptide, Biochem J 421, 415-423.

[68] Sun, Y., Kakinen, A., Wan, X., Moriarty, N., Hunt, C. P. J., Li, Y., Andrikopoulos, N., Nandakumar, A., Davis, T. P., Parish, C. L., Song, Y., Ke, P. C., and Ding, F. (2021) Spontaneous formation of $\beta$-sheet nano-barrels during the early aggregation of Alzheimer's amyloid beta, Nano Today 38, 101125.

[69] Abedini, A., Plesner, A., Cao, P., Ridgway, Z., Zhang, J., Tu, L.-H., Middleton, C. T., Chao, B., Sartori, D. J., Meng, F., Wang, H., Wong, A. G., Zanni, M. T., Verchere, C. B., Raleigh, D. P., and Schmidt, A. M. (2016) Time-resolved studies define the nature of toxic IAPP intermediates, providing insight for anti-amyloidosis therapeutics, eLife 5, e12977.

[70] Berendsen, H., Postma, J. P. M., van Gunsteren, W., and Hermans, J. (1981) Interaction Models for Water in Relation to Protein Hydration, pp 331-342.

[71] Foloppe, N., and MacKerell, J., Alexander D. (2000) All-atom empirical force field for nucleic acids: I. Parameter optimization based on small molecule and condensed phase macromolecular target data, Journal of computational chemistry 21, 86-104.

[72] Gerben, S. R., Lemkul, J. A., Brown, A. M., and Bevan, D. R. (2014) Comparing atomistic molecular mechanics force fields for a difficult target: a case study on the Alzheimer's amyloid $\beta$-peptide, Journal of Biomolecular Structure and Dynamics 32, 1817-1832.

[73] Ahmed, M. C., Papaleo, E., and Lindorff-Larsen, K. (2018) How well do force fields capture the strength of salt bridges in proteins?, PeerJ 6, e4967. 